\title{
Challenges towards the realization of individualized cancer vaccines
}

\author{
Bringing truly personalized cancer vaccination with tumour neoantigens to the clinic will require overcoming the \\ challenges of optimized vaccine design, manufacturing and affordability, and identification of the most suitable \\ clinical setting.
}

\section{Özlem Türeci, Martin Löwer, Barbara Schrörs, Maren Lang, Arbel Tadmor and Ugur Sahin}

mmunotherapies can achieve durable
clinical responses in patients with
advanced cancers that are unresponsive
to the current standard of care. In a
considerable number of patients receiving
adoptive transfer of autologous melanoma-
reactive tumour infiltrating lymphocytes
(TILs), tumour regressions have been
observed. These correlate with TIL reactivity
against 'neo-epitopes' derived from somatic
cancer mutations ${ }^{1}$. Antibodies blocking the
immune checkpoint molecules CTLA-4
(cytotoxic T-lymphocyte-associated antigen
4 ) and PD-1/PD-L1 (programmed cell
death protein 1 and programmed cell death
ligand 1, respectively) have been approved
for clinical use following demonstration
of long-term survival in a proportion of
patients across a variety of cancer types ${ }^{2,3}$.
This immunotherapy strategy, broadly
referred to as checkpoint inhibition or referred to as checkpoint inhibition or checkpoint blockade, unleashes suppressed T cells capable of recognizing and killing cancer cells. Several studies have shown that $\mathrm{T}$ cells whose activity is restored by checkpoint inhibition also target cancer mutations ${ }^{4}$. Accordingly, the clinical success of treatment with checkpoint inhibitors is superior in patients with higher mutational $\operatorname{load}^{5}$. However, cancer patients have spontaneous T-cell immunity against only a small fraction $(<1 \%)$ of their mutations ${ }^{6}$. Broadening this tumour-reactive T-cell repertoire may be achieved by vaccination with cancer mutations (Fig. 1).

Recent first-in-human clinical trials, in patients with malignant melanoma, suggest that vaccination with such neo-epitopes may lead to the realization of a truly personalized cancer treatment. Engineering a vaccine tailored to the individual genetic make-up of a patient's tumour to mobilize their immune system is a tantalizing concept. The idea of customizing therapy is of course not new, yet the development of personalized medicines only recently became feasible by disruptive breakthroughs in interconnected areas of technology and science. Indeed,

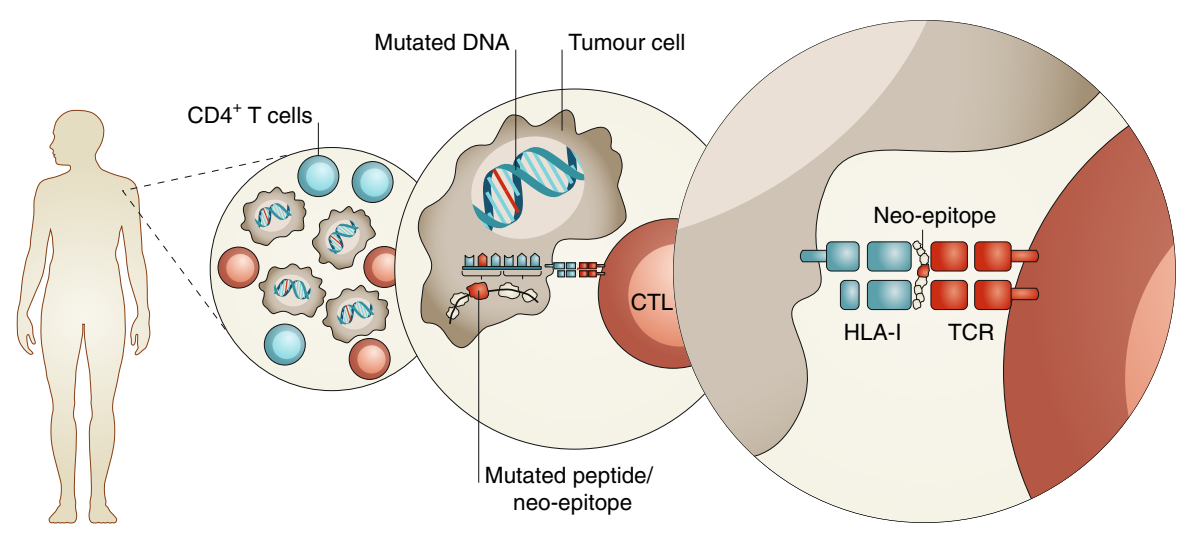

Fig. 1 | Recognition of cancer-associated mutations by cytotoxic T cells. Cancer cells harbour genomic aberrations. A fraction of these mutations alter the sequence of expressed proteins, which are then processed and presented on the HLA class-I molecules of tumour cells. These molecules may be recognized by $C D 8^{+}$cytotoxic $T$ lymphocytes (CTLs) as foreign neo-epitopes, and may, with $C D 4^{+}{ }^{+}$-cell help, trigger an immune response that results in tumour-cell-specific killing. TCR, T cell receptor.

major progress in improving the speed, cost and quality of sequencing, combined with bioinformatics tools, advances in highthroughput manufacturing and a deeper understanding of the immune system, have all converged to make individualized therapies a reality.

\section{Individualized neo-epitope vaccines}

Systematic testing of vaccination with peptides derived from somatic cancer mutations, which were identified by nextgeneration sequencing (NGS) in syngeneic mouse tumour models, revealed that a substantial fraction $(20 \%-50 \%)$ of these mutations is immunogenic and exhibits potent therapeutic activity ${ }^{7}$. Several other reports independently confirmed that vaccines composed of computationally predicted neo-epitopes are capable of exhibiting strong anti-tumour activity and can mediate rejection of established mouse tumours ${ }^{8-10}$. In human cancer, however, every patient has an exquisitely individual mutational signature, with only a very small fraction of mutations shared between patients. Thus, only by a personalized approach can the full spectrum of mutations displayed by the tumour of a given patient be leveraged to mobilize broad and polyclonal anti-tumour immunity ${ }^{11}$.

Clinical application of neo-epitope vaccines requires profiling of each patient's tumour by NGS, followed by computational prediction of neo-antigens and selection of those to be incorporated into a vaccine of unique composition, which is then produced on-demand. At first glance, engineering a personal drug for each and every individual seems to be incompatible with the contemporary 'off-the-shelf' paradigm of drug development and clinical practice; yet this goal has been achieved by recent efforts integrating substantial technical, coordinative and multidisciplinary skills.

In 2015, a first step towards vaccination with individual neo-antigens was attained by the treatment of three melanoma patients using autologous dendritic cells loaded with a personalized mixture of 




Fig. 2 | Workflow for the development of cancer neo-epitope vaccines for personalizing immunotherapy. Tumour biopsies and healthy tissue (for example, peripheral blood cells) of a patient diagnosed with cancer are subjected to NGS. Tumour-specific mutations in protein-coding genes are identified by comparing sequences obtained from tumour and normal DNA. The mutant peptides encoded by these altered DNA sequences are then processed by algorithms that predict whether they would bind to one of the patient's HLA alleles. Additional features of the mutated protein deemed to be potential determinants for clinically meaningful vaccine responses also inform the selection of mutations for the design of a multi-epitope vaccine. A vaccine of unique composition is then produced for each and every patient under good manufacturing practice.

seven 9-mer peptides predicted to bind to human leukocyte antigen (HLA)-A2, the most frequent HLA class-I haplotype shared by the three patients ${ }^{12}$. Vaccineinduced $\mathrm{CD}^{+} \mathrm{T}$ cells against 9 of the 21 mutated peptides used across patients were detected, and their specificity against the respective immunogens confirmed. In 2017, two studies reported the vaccination of patients with neo-epitopes predicted for each patient's personal set of HLA haplotypes, thereby actualizing the full potential of individualization ${ }^{13,14}$. Despite the small sample sizes ( 6 patients in ref. ${ }^{13}$ and 13 patients in ref. ${ }^{14}$, all with advanced malignant melanoma), differences in the computational pipelines used for selecting the mutated targets, and application of different vaccine platforms, these studies reported similar findings. In both studies, strong $\mathrm{CD}^{+}$and $\mathrm{CD} 8^{+} \mathrm{T}$-cell reactivity was induced in every patient against several mutated epitopes, which led to the expansion of both pre-existing $\mathrm{T}$ cells as well as to the induction of de novo T-cell responses against neo-epitopes not recognized prior to vaccination. Moreover, both studies provided initial evidence of clinical activity, with objective responses observed with the vaccine alone and in combination with subsequent checkpoint-inhibition treatment. Both vaccines were safe and well-tolerated. These studies have overcome the critical hurdles of early clinical translation, and will inspire further clinical exploration of personalized neo-epitope vaccination towards the realization of its full potential.

\section{Challenges ahead}

The generic workflow for individualized neo-epitope vaccination consists of several steps (Fig. 2). Whole exome and RNA sequencing data is obtained by NGS analyses of the patient's tumour tissue and healthy cells (the latter derived, in general, from circulating blood cells). Computational tools are then employed to compare data from these matched samples, identify cancer-specific mutations and quantify the expression of each mutated gene. Next, mutations are ranked primarily on the basis of the predicted binding affinity of the putative epitopes to the patient's HLA alleles and their expression, while factoring in the clonality of the respective mutation. The top rankers are then used for subsequent vaccine design. For each patient, a unique vaccine comprised of multiple predicted neo-epitopes is manufactured, individually released and administered. The first-inhuman trials have shown that integration and quality control of the entire process is feasible and compatible with the general practices of clinical testing in a healthcare setting.

The next key issues that need to be addressed for the further development of personalized cancer vaccines are (i) optimization of the vaccine for its immunepharmacological purpose; (ii) up-scaling of manufacturing while ensuring affordability; and (iii) identification of the most suitable clinical indications and treatment protocols (Fig. 3). We will discuss each of these issues separately.

\section{Vaccine composition and delivery}

Neo-epitopes are ideal cancer vaccine targets, as they are exempt from central immune tolerance and absent from healthy tissue, hence providing an excellent immunogenicity and safety profile. However, realization of the full therapeutic potential of neo-epitope vaccines depends on their overall composition and delivery.

It is desirable for a cancer vaccine to induce a diverse repertoire of both $\mathrm{CD} 4^{+}$ and $\mathrm{CD} 8^{+} \mathrm{T}$ cells directed against a range of neo-epitopes, preferably representing to some extent the clonal heterogeneity of the tumour. Bundling multiple neo-epitopes in the same vaccine enhances the effectiveness of the T-cell response and minimizes the risk of outgrowth of antigen-loss tumour-cell variants. The optimal number of different mutations a vaccine should feature is not yet known, and may depend on the mutational load and clonality pattern $n^{5}$ of the respective tumour.

Sensitive detection and proper selection of the most suitable vaccine targets is of crucial importance. Mutation detection on an exome scale should strive to be robust to the quality, amount and tumour-content of the provided biopsy. Tumours with high stromal contamination are a challenge for the sensitivity and specificity of mutation calls ${ }^{15}$. Single-nucleotide substitutions, which are the most frequent mutations and technically easier to identify, and to a lesser extent insertions or deletions of one or a few bases (indels), are currently used for neo-epitope vaccines. For robust identification of the various other categories of cancer-associated genetic aberrations (such as frameshift indels, gene fusions and open reading frames (ORFs) generated by gene fusions), computational tools need to be further improved. Moreover, mutation calling is currently restricted to established protein sequences, as the large 'non-coding' part of the genome, and cancer-specifically translated non-canonical ORFs, are not 


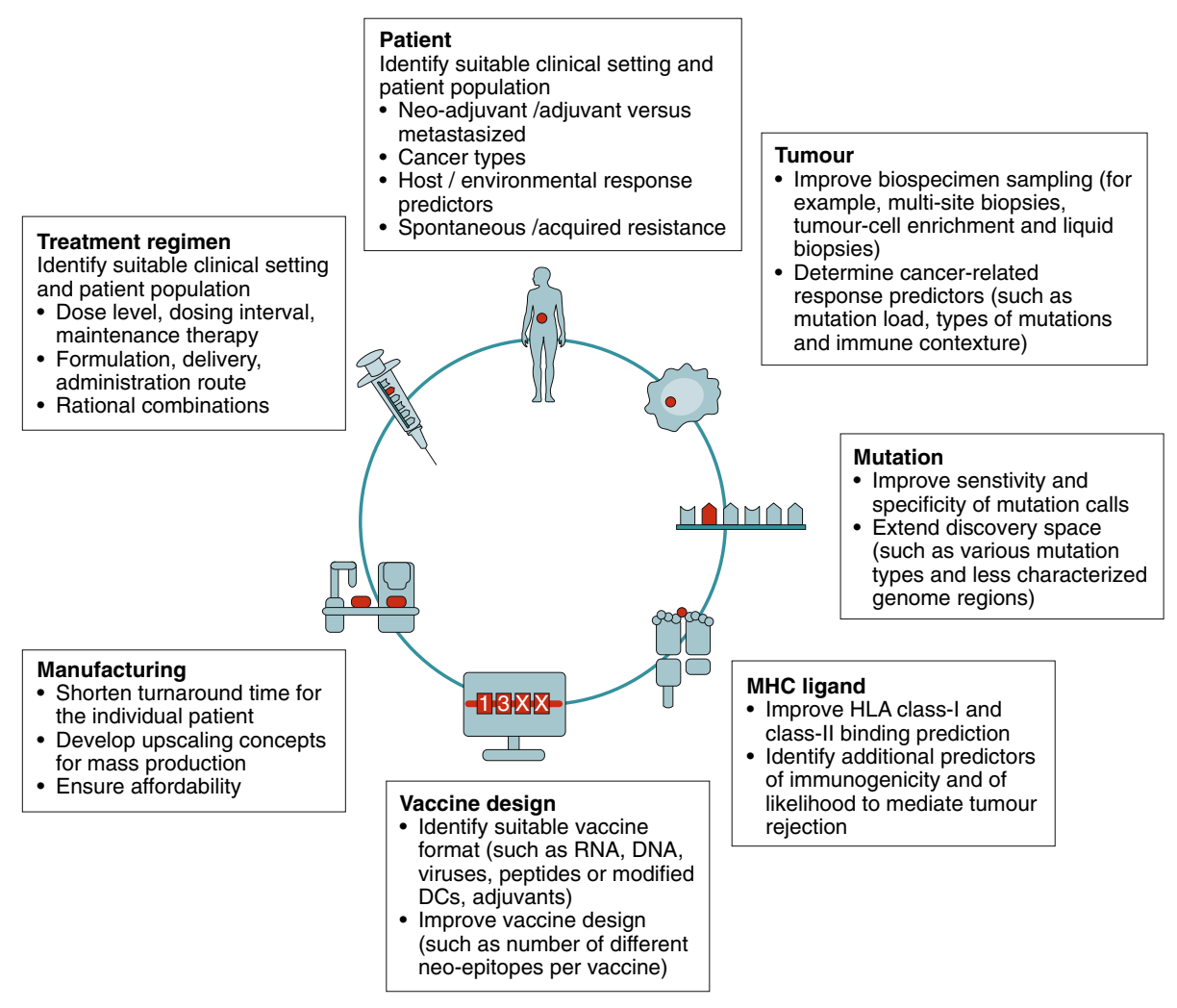

Fig. 3 | Key challenges for actualizing the concept of personalized neo-epitope cancer vaccines.

Translating neo-epitope vaccines into mainstream clinical oncology is going to require highly interdisciplinary solutions. DC, dendritic cells; MHC, major histocompatibility complex.

considered $^{16}$. The study of these uncharted genomic regions may help to serve patients with low tumour mutational load and to unravel types of genetic aberrations that are better suited as immunogens for certain tumour types (as shown for renal cell cancer, in which indels seem to be highly enriched and immunogenic ${ }^{17}$ ).

The prediction of mutations with the highest likelihood of immunogenicity and therapeutic impact is an immediate challenge. Current efforts rely mostly on predicting the binding affinity to patients' HLA molecules. Many other factors beyond this elementary prerequisite for immunogenicity (in particular, the likelihood of recognition by T-cell receptors) are not considered. Yet in the two recent clinical studies, about $60 \%$ of the mutations selected as vaccine targets exhibited a T-cell response, most of which were de novo induced. Overall, this is an excellent immunogenicity rate for purely computationally predicted neoepitopes in an unbiased patient population. Nevertheless, it is expected that not every mutation that is immunogenic will ultimately confer anti-tumour activity. It is also probable that not all clinically relevant neo-epitopes can be identified by current prediction tools. Therefore, refinement of computational tools for prediction of the affinity and stability of binding to HLA molecules (including rare HLA class-I and HLA class-II haplotypes) and understanding of other potential predictors of immunogenicity (such as sequence similarity to microbial HLA epitopes) are ongoing efforts toward the optimization of vaccine-target selection ${ }^{18,19}$.

Further improvement of the specificity and sensitivity of choosing the right mutations will be achieved by the development of a broader database, generated from clinical neo-epitope trials, mutanome studies in cancer patient cohorts, and mass-spectrometry-assisted HLA ligandome analyses performed in conjunction with machine learning ${ }^{20-22}$.

Identification of suitable vaccine targets is essential but not sufficient for clinical success. One needs to ensure that targeted antigens are efficiently presented on dendritic cells under conditions that overcome immune suppression and result in strong, diversified T-cell responses and immunological memory ${ }^{23}$. This requires a potent vaccine system. Various vaccine formats - including mRNA, DNA, synthetic peptides, recombinant viruses and adoptive transfer of modified dendritic cells - are currently being tested for neoepitope delivery. Even for conventional off-the-shelf cancer vaccines, there is no pharmaceutically validated technology yet. The lack of an established technology can be a blessing in disguise, as it gives full freedom to factor-in personalization-specific challenges early on in the selection of the most suitable vaccine format.

\section{Manufacturing and affordability}

By dictating speed, scalability and costs, manufacturing is probably the most critical element for the viable implementation of personalized vaccines into clinical practice and the corresponding commercial landscape. A vaccine format that is synthetic and allows for fast production at low cost by an unsophisticated, robust, invariant and GMP (good manufacturing practice)compliant process, will therefore be essential.

The two recently reported clinical trials ${ }^{13,14}$ used either a mixture of long peptides featuring the selected mutations with the immunostimulant poly-ICLC as adjuvant, or mRNA with its intrinsic adjuvant activity encoding fusion peptides comprising the mutated sequences. In these trials, turnaround times (from processing the patient's sample to release of the drug product) were about three months. The interval between patient enrolment and vaccine administration can be bridged by treating patients with other drugs. For both vaccine systems, it is anticipated that the time-to-delivery will be reduced to less than four weeks.

Manufacturing customized cancer vaccines for the mass market cannot be achieved by upscaling production volumes. Instead, the goal is to increase the number of production campaigns per time, with each campaign representing a drug product for one individual. The realization of this approach requires innovative costoptimized and time-optimized production technologies. Such concepts are in sync with contemporary trends in manufacturing that are triggered by breakthroughs in other areas that are personalized themselves, for example, in autologous cell therapy at the industrial scale ${ }^{24}$. Moreover, the convergence of advances in computational power, connectivity, analytics, humanmachine interactions, robotics (as enablers of full digitization of production processes and of autonomous cloud-controlled production plants) and $3 \mathrm{D}$ printing (for building massively parallelized, miniaturized production lines) - collectively referred to as the fourth industrial revolution ${ }^{25}-$ is expected to provide solutions for the mass production of customized products. 
Drug affordability is a frequently raised topic in the context of innovative drugs, including personalized medicine. Generally speaking, the primary concern is not the drug price but whether the therapeutic returns justify a typically substantial price. The loftier goal of personalized vaccination is to give every cancer patient the best chance of addressing their 'one-of-a-kind' cancer, which may harmonize with the trend towards outcome-based and value-based pricing and reimbursement models.

\section{Clinical development}

The path forward for clinical development of personalized vaccines does not differ much from that of off-the-shelf drugs, and involves comparison to the standard of care in randomized studies. However, personalized vaccine development carries peculiar challenges $^{26}$. One pertinent question is which clinical setting would be most appropriate for a neo-epitope cancer vaccine. The adjuvant setting and minimal residual disease have the advantage that immune-suppressive mechanisms and stromal factors are not firmly established. However, it is unclear whether vaccine-induced $\mathrm{T}$ cells are the primary mediators of the therapeutic effect, or rather serve as initiators to mobilize a broader T-cell repertoire by selfpropagating cycles of antigen spread ${ }^{27}$. The latter scenario would require the presence of bulk tumour tissue at the time of vaccination and would favour metastatic disease. Furthermore, the treatment of cancer patients with locally advanced disease carries the risk that distant micrometastases may clonally differ from the resected primary tumour that informed the composition of the vaccine. Conversely, in progressing metastatic disease the turnaround time of a vaccine may become a limiting factor. Moreover, higher intratumoural and interlesional heterogeneity, as well as advanced immune suppression and escape mechanisms, may pose biological hurdles.
Neo-epitope vaccines seem to be safe and well-tolerated, which is in line with the notion that they target strictly cancerspecific epitopes. Thus, combining them with other drugs carries a low risk of added toxicities while allowing the advantage of synergistic modes of action. There is a strong rationale for the combination of neo-epitope vaccination with checkpoint inhibition to keep the expanded repertoire of vaccine-induced T-cell specificities functional. The obstacle of an immuneexcluding or immune-suppressive tumour microenvironment can be overcome by combining the vaccine with treatment modalities that counteract these phenomena (such as inhibitors of indoleamine 2,3-dioxygenase or of colony stimulating factor- 1 receptor $)^{28}$. The most probable escape mechanisms from a strong, multiantigen T-cell response involve loss of the target antigen or antigen-presentation machinery. This can be addressed by combining the vaccine with immuneeffectors, such as chimaeric antigen receptor (CAR)-engineered T cells or bispecific T-cell engagers, which do not depend on HLApresented antigens.

\section{Outlook}

In conclusion, perhaps not surprisingly for a breakthrough innovation, translating neo-epitope vaccines into mainstream clinical oncology will require highly interdisciplinary solutions and an innovative drug-development process that takes advantage of the integration of automation and digitalization technologies. Mutations are a hallmark of cancer and the basis for its intrinsic heterogeneity and spatiotemporal plasticity. They are associated with the failure of contemporary treatment approaches. Mutation-based vaccines will thus make bona fide biology-guided drugs that are agnostic to the cancer type and universally applicable to any patient.

Özlem Türeci', Martin Löwer², Barbara Schrörs ${ }^{2}$, Maren Lang ${ }^{3}$, Arbel Tadmor ${ }^{2}$ and Ugur Sahin ${ }^{2,3,4 \star}$
${ }^{1}$ Cluster for Individualized Immune Intervention (CI3), Mainz, Germany. ${ }^{2}$ TRON-Translational Oncology at the University Medical Center of Johannes Gutenberg University gGmbH, Mainz, Germany. ${ }^{3}$ Biopharmaceutical New Technologies (BioNTech) Corporation, Mainz, Germany. ${ }^{4}$ University Medical Center of the Johannes Gutenberg University, Mainz, Germany.

*e-mail:sahin@uni-mainz.de

Published online: 30 July 2018

https://doi.org/10.1038/s41551-018-0266-2

References

1. Robbins, P. F. et al. Nat. Med. 19, 747-752 (2013).

2. Pardoll, D. M. Nat. Rev. Cancer 12, 252-264 (2012).

3. Sharma, P. \& Allison, J. P. Science 348, 56-61 (2015).

4. Schumacher, T. N. \& Schreiber, R. D. Science 348, 69-74 (2015)

5. Mcgranahan, N. et al. Science 351, 1463-1469 (2016).

6. Tran, E. et al. Science 350, 1387-1390 (2015).

7. Castle, J. C. et al. Cancer Res. 72, 1081-1091 (2012).

8. Kreiter, S. et al. Nature 520, 692-696 (2015).

9. Gubin, M. M. et al. Nature 515, 577-581 (2014).

10. Yadav, M. et al. Nature 515, 572-576 (2014).

11. Kreiter, S., Castle, J. C., Türeci, O. \& Sahin, U. OncoImmunology 1, 768-769 (2012).

12. Carreno, B. M. et al. Science 348, 803-808 (2015)

13. Ott, P. A. et al. Nature 547, 217-221 (2017).

14. Sahin, U. et al. Nature 547, 222-226 (2017).

15. Löwer, M. et al. PLoS Comput. Biol. 8, e1002714 (2012).

16. Laumont, C. M. \& Perreault, C. Cell. Mol. Life Sci. 75, 607-621 (2017)

17. Turajlic, S. et al. Lancet Oncol. 18, 1009-1021 (2017).

18. Balachandran, V. P. et al. Nature 551, 512-516 (2017).

19. Łuksza, M. et al. Nature 551, 517-520 (2017).

20. Khodadoust, M. S. et al. Nature 543, 723-727 (2017).

21. Abelin, J. G. et al. Immunity 46, 315-326 (2017).

22. Luo, H. et al. Bioinform. Biol. Insights https://doi.org/10.4137/BBI \$29466 (2015).

23. Kranz, L. M. et al. Nature 534, 396-401 (2016).

24. Harrison, R. P., Ruck, S., Rafiq, Q. A. \& Medcalf, N. Biotechnol. Adv. 36, 345-357 (2018).

25. Kagermann, H. in Management of Permanent Change 23-45 (Springer Fachmedien Wiesbaden, 2015).

26. Türeci, O. et al. Clin. Cancer Res. 22, 1885-1896 (2016).

27. Chen, D. S. \& Mellman, I. Nature 541, 321-330 (2017) 28. Melero, I. et al. Nat. Rev. Cancer 15, 457-72 (2015).

\section{Competing interests}

U.S. and M.La. are employees at BioNTech AG (Mainz, Germany), a privately owned company developing individualized immunotherapies. U.S. is co-founder, stock owner and management board member of BioNTech AG. Ö.T., M.Lö., B.S., M.La., A.T. and U.S. are inventors on patents and patent applications, which are related to some of the technologies described in this article. 